\title{
ON WATSON'S QUINTUPLE PRODUCT IDENTITY
}

\author{
M. V. SUBBARAO ${ }^{1}$ AND M. VIDYASAGAR ${ }^{2}$
}

ABSTRACT. In 1929, in the course of proving certain results stated by Ramanujan concerning his continued fraction, G. N. Watson proved an identity involving five infinite products and an infinite series. In 1938, Watson proved another identity which again involved five products. Finally in 1961, one more quintuple product identity was established, this time by Basil Gordon. We show here that all these identities are equivalent. Also, with the help of the quintuple product identity and Jacobi's triple product identity, we establish two new identities involving only series.

1. The identities of Watson and Gordon. In 1961 Basil Gordon [2] proved the following identity:

$$
\begin{aligned}
\prod_{n=1}^{\infty}\left(1-s^{n}\right)(1 & \left.-s^{n} t\right)\left(1-s^{n-1} t^{-1}\right)\left(1-s^{2 n-1} t^{2}\right)\left(1-s^{2 n-1} t^{-2}\right) \\
& =\sum_{-\infty}^{\infty} s^{\left(3 n^{2}+n\right) / 2}\left(t^{3 n}-t^{-3 n-1}\right), \quad|s|<1, t \neq 0 .
\end{aligned}
$$

It does not seem to have been noticed that Gordon was anticipated by G. N. Watson [3, pp. 44-45] who proved the identity in 1929 in the following equivalent form using essentially the same argument as Gordon's (see line 12 on p. 45 of [3]):

$$
\begin{aligned}
\sum_{n=1}^{\infty}\left(1-q^{5 n}\right)\left(1+q^{5 n-1} z^{-1}\right)\left(1+q^{5 n-4} z\right)\left(1-q^{10 n-7} z^{-2}\right)\left(1-q^{10 n-3} z^{2}\right) \\
=\sum_{n=-\infty}^{\infty}(-1)^{n} q^{m(15 m+1) / 2} z^{3 m}+\sum_{n=-\infty}^{\infty}(-1)^{m} q^{(5 n-2)(3 n-1) / 2} z^{1-3 n} .
\end{aligned}
$$

In 1938, Watson [4] gave also the following identity (we here change Watson's notation):

Received by the editors January $5,1970$.

AMS 1969 subject classifications. Primary 1048; Secondary 1055, 0510.

Key words and phrases. Identities of Euler, Jacobi's triple product identity, quintuple product identities of Basil Gordon.

1 The work of the first author was supported in part by the National Research Council of Canada Grant No. A-3103.

2 Present address: Sir George Williams University, Montreal, Canada. 


$$
\begin{aligned}
& \prod_{n=1}^{\infty} \frac{\left(1-x^{2 n}\right)\left(1-a^{2} x^{2 n-2}\right)\left(1-a^{-2} x^{2 n}\right)}{\left(1+a x^{2 n-1}\right)\left(1+a^{-1} x^{2 n-1}\right)} \\
& \quad=\sum_{n=-\infty}^{\infty}\left(a^{-3 n}-a^{3 n+2}\right) x^{n(3 n+2)}, \quad|x|<1, a \neq 0 .
\end{aligned}
$$

We wish to point out that this identity is equivalent to (1.1) and (1.2). The purpose of this paper is, first, to give a formal proof of this statement, and next, to prove, in $\$ 3$, the following two striking identities involving only series, which are valid-like everything else that follows-for $|x|<1$ and $a \neq 0$ :

$$
\begin{array}{r}
\sum_{n=1}^{\infty}(-1)^{n-1} a^{n-1} x^{n-1}(1+a x) \\
\left(1+a x^{3}\right)\left(1+a x^{5}\right) \cdots\left(1+a x^{2 n-3}\right) \\
=1+\sum_{n=1}^{\infty} a^{3 n-1} x^{3 n^{2}}\left(a x^{2 n}-a^{-1} x^{-2 n}\right)
\end{array}
$$

$$
\begin{gathered}
\sum_{n=1}^{\infty} \frac{(-1)^{n-1} a^{2 n-2} x^{n(n-1)}}{(1+a x)\left(1+a x^{3}\right)\left(1+a x^{5}\right) \cdots\left(1+a x^{2 n-1}\right)} \\
=1+\sum_{n=1}^{\infty} a^{3 n-1} x^{3 n^{2}}\left(a x^{2 n}-a^{-1} x^{-2 n}\right) .
\end{gathered}
$$

We shall derive these results with the help of identity (1.3) - which we shall henceforth designate as (Watson's) Quintuple Product Identity - and the well-known Triple Product Identity of Jacobi [1]:

$$
\prod_{n=1}^{\infty}\left(1-x^{2 n}\right)\left(1+a x^{2 n-1}\right)\left(1+a^{-1} x^{2 n-1}\right)=\sum_{n=-\infty}^{\infty} a^{n} x^{n^{2}} .
$$

Conversely, we shall show that identities (1.4) and (1.5) together with the Triple Product Identity would imply Watson's Quintuple Product Identity.

2. The equivalence of Gordon's and Watson's identities. We shall prove the equivalence of (1.1) and (1.3). On using the obvious relation

$$
\begin{aligned}
\prod_{n=1}^{\infty}\left(1-a^{2} x^{2 n-2}\right) & =\prod_{n=1}^{\infty}\left(1-a^{2} x^{4 n-2}\right)\left(1-a^{2} x^{4 n-4}\right) \\
& =\prod_{n=1}^{\infty}\left(1-a x^{2 n-1}\right)\left(1+a x^{2 n-1}\right)\left(1-a^{2} x^{4 n-4}\right),
\end{aligned}
$$

and a similar relation for $\prod_{n=1}^{\infty}\left(1-a^{-2} x^{2 n}\right)$, the identity (1.3) becomes 


$$
\begin{gathered}
\prod_{n=1}^{\infty}\left(1-x^{2 n}\right)\left(1-a x^{2 n-1}\right)\left(1-a^{-1} x^{2 n-1}\right)\left(1-a^{2} x^{4 n-4}\right)\left(1-a^{-2} x^{4 n}\right) \\
=\sum_{n=-\infty}^{\infty}\left(a^{-3 n}-a^{3 n+2}\right) x^{n(3 n+2)} .
\end{gathered}
$$

Substituting $s=x^{2}, t=a x^{-1}$ in (2.1), we get

$$
\begin{aligned}
\prod_{n=1}^{\infty}\left(1-s^{n}\right)\left(1-t s^{n}\right) & \left(1-t^{-1} s^{n-1}\right)\left(1-t^{2} s^{2 n-1}\right)\left(1-t^{-2} s^{2 n-1}\right) \\
= & \sum_{n=-\infty}^{\infty} s^{n(3 n+2) / 2}\left(t^{3 n} s^{-3 n / 2}-t^{3 n+2} s^{3 n / 2+1}\right) \\
= & \sum_{n=-\infty}^{\infty} s^{3 n^{2} / 2-n / 2} t^{-3 n}-\sum_{n=-\infty}^{\infty} s^{3 n^{2} / 2+5 n / 2+1} t^{3 n+2}
\end{aligned}
$$

In the first summation, replace $n$ by $-m$, while in the second, replace $n$ by $-m-1$. We get for the right side of (2.2):

$$
\sum_{m=-\infty}^{\infty} s^{3 m^{2} / 2+m / 2 t^{3 m}}-\sum_{m=-\infty}^{\infty} s^{3 m^{2} / 2+m / 2 t-3 m-1}=\sum_{m=-\infty}^{\infty} s^{\left(3 m^{2}+m\right) / 2}\left(t^{3 m}-t^{-3 m-1}\right) .
$$

This shows that (1.3) is equivalent to (1.1).

3. An identity between series. We shall now prove the

THEOREM.

$$
\begin{aligned}
& \sum_{n=1}^{\infty}(-1)^{n} a^{n+1} x^{n-1}(1+a x)\left(1+a x^{3}\right) \cdots\left(1+a x^{2 n-3}\right) \\
& +\sum_{n=1}^{\infty}(-1)^{n-1} a^{2-2 n} x^{n(n-1)}\left(1+a^{-1} x\right)^{-1}\left(1+a^{-1} x^{3}\right)^{-1} \\
& \cdots\left(1+a^{-1} x^{2 n-1}\right)^{-1} \\
& \quad=\sum_{n=-\infty}^{\infty}\left(a^{-3 n}-a^{3 n+2}\right) x^{3 n^{2}+2 n} .
\end{aligned}
$$

Proof. In addition to Watson's Quintuple Product Identity (1.3) and Jacobi's Triple Product Identity (1.6), we require the well-known Euler identities [1]:

$$
\prod_{n=1}^{\infty}\left(1-a x^{n}\right)=\sum_{n=0}^{\infty} \frac{(-1)^{n} a^{n} x^{n(n+1) / 2}}{(1-x)\left(1-x^{2}\right) \cdots\left(1-x^{n}\right)},
$$




$$
\frac{1}{\prod_{n=1}^{\infty}\left(1-a x^{n}\right)}=\sum_{n=0}^{\infty} \frac{a^{n} x^{n}}{(1-x)\left(1-x^{2}\right) \cdots\left(1-x^{n}\right)} .
$$

Let

$$
\phi_{n}(a, x)=(1-a x)\left(1-a x^{2}\right) \cdots\left(1-a x^{n}\right), \quad n>0,
$$

with $\phi_{0}(a, x)=1$ and $\phi_{n}(x)=\phi_{n}(1, x)$.

Applying (1.3), (1.6) and (3.3), we have

$$
\begin{aligned}
\prod_{n=1}^{\infty}(1+ & \left.a^{-1} x^{2 n-1}\right) \sum_{n=-\infty}^{\infty}\left(a^{-3 n}-a^{3 n+2}\right) x^{3 n^{2}+2 n} \\
& =\frac{1}{\prod_{n=1}^{\infty}\left(1+a x^{2 n-1}\right)^{n=1}} \prod^{\infty}\left(1-x^{2 n}\right)\left(1-a^{2} x^{2 n-2}\right)\left(1-a^{-2} x^{2 n}\right) \\
& =\sum_{m=0}^{\infty} \frac{(-1)^{m}\left(a x^{-1}\right)^{m} x^{2 m}}{\phi_{m}\left(x^{2}\right)}\left(\sum_{n=-\infty}^{\infty}(-1)^{n}\left(a^{2} x^{-1}\right)^{n} x^{n^{2}}\right)
\end{aligned}
$$

Writing $n-m$ for $n$ in the inner sum, we see that (3.5) is the same as

$$
\sum_{m=0}^{\infty} \sum_{n=-\infty}^{\infty}(-1)^{n} x^{n^{2}-n+m^{2}-2 m n+2 m} a^{2 n-m} / \phi_{m}\left(x^{2}\right) .
$$

Recalling the assumption made throughout that $|x|<1$ and $a \neq 0$, the series (3.6) is absolutely convergent and rearrangement of its terms is permitted. Therefore, (3.6) can be written as

$$
\sum_{n=-\infty}^{\infty}(-1)^{n} x^{n^{2}-n} a^{2 n} \sum_{m=0}^{\infty} \frac{x^{m^{2}-2 m n+2 m} a^{-m}}{\phi_{m}\left(x^{2}\right)} .
$$

On using (3.2) for the inner sum, we get

$$
\sum_{n=-\infty}^{\infty}(-1)^{n} x^{n^{2}-n} a^{2 n} \prod_{r=1}^{\infty}\left(1+\frac{x^{1-2 n}}{a}\right) x^{2 r} .
$$

On carrying out some routine manipulations, this can be written as $\psi(a, x) \prod_{r=1}^{\infty}\left(1+a^{-1} x^{2 r-1}\right)$ where $\psi(a, x)$ is the left side in the relation (3.1). This completes the proof of (3.1).

(3.8) Proof of (1.4) AND (1.5). On equating those expressions on both sides of (3.1) which consist entirely of terms with positive powers of $a$, we obtain (1.4) after some simplification. Observe that for the left side of (3.1), this expression is exactly the first sum there. To 
obtain (1.5), we proceed similarly taking only terms with negative powers of $a$ and afterwards replace $a$ by $a^{-1}$.

4. Remarks. The identities (1.4) and (1.5) arose in our attempt for a simple proof of Watson's Quintuple Product Identity (1.3) on the same lines as G. E. Andrews' proof [1] of Jacobi's Triple Product Identity. If the results (1.4) and (1.5) are already known (though we are unable to locate them in the literature), or if they can be proved easily otherwise, the proof of Theorem (3.1) provides a simple alternate proof of (1.3) different from Watson's or Gordon's. On the other hand, if the results (1.4) and (1.5) are new, it would be surprising how one missed such beautiful identities for so long!

\section{REFERENCES}

1. G. E. Andrews, $A$ simple proof of Jacobi's triple product identity, Proc. Amer. Math. Soc. 16 (1965), 333-334. MR 30 \#1952.

2. B. Gordon, Some identities in combinatorial analysis, Quart. J. Math. Oxford Ser. (2) 12 (1961), 285-290. MR 25 \#21.

3. G. N. Watson, Theorems stated by Ramanujan (VII): Theorems on continued fractions, J. London Math. Soc. 4 (1929), 39-48.

4. - Ramanujan's Vertumung über Zerfallungsanzahlen, J. Reine Angew. Math. 179 (1938), 97-128.

University of Alberta, Edmonton, Alberta, Canada

Marquette University, Milwaukee, Wisconsin 53233 